\title{
Equivalent Gibbs Duhem Law in Network Queue
}

\author{
Sandeep Sudeep and Joseph Y.Hui \\ Sandeep Sudeep, Electrical Engineering, Arizona State University, \\ Tempe, AZ 85287 USA \\ J. Hui, Electrical Engineering, Arizona State University, \\ Tempe, AZ 85287 USA \\ sandeepsudeep@asu.edu, jhui@asu.edu
}

\begin{abstract}
High speed networks are highly dynamical systems. Large deviation techniques have been employed to characterize their loss probability. Large deviation is also used to calculate shadow prices of quality of service of a scalable network queue with respect to buffer size $\mathrm{B}$, capacity $\mathrm{C}$ and arrival rate N.In this paper we prove that these shadow prices are independent of $(B, C, N)$ parameters. Gibbs Duhem law of thermodynamics is described. Equivalent Gibbs Duhem type law in scalable network queue is derived. Application of equivalent network Gibbs Duhem law is discussed.
\end{abstract}

Index Terms: Quality of Service, Macroscopic parameter, Microscopic Parameter, Gibbs Duhem law.

\section{Introduction}

Quality of service of a network queue is defined as negative logarithm of its loss probability. Loss probability in high speed network is usually defined in large deviation regime. Hui[1] proves the linear scalability of quality of service(Q) of a network queue with respect to capacity $(\mathrm{C})$, number of connections(N) and buffer size(B).Hui[1] defines microscopic and macroscopic entities in a scalable queue equivalent to microscopic and macroscopic parameters in a thermodynamic system. Buffer B, Capacity $\mathrm{C}$, arrival rate $\mathrm{N}$ and quality of service $\mathrm{Q}$ are defined as macroscopic properties of queue whereas rate of change of one macroscopic parameters with other is defined as microscopic parameter.

In this paper we prove that microscopic parameters of a scalable network queue are related to each other independent of macroscopic parameters of network queue. We describe Gibbs Duhem law in thermodynamics and present equivalent Gibbs Duhem law in a scalable network queue. Gibbs Duhem law relates microscopic entities of a network queue in differential form.

Section 2 presents a brief introduction to Hui[1]. Section 3 presents relationship between microscopic entities. Section 4 discusses Gibbs Duhem law of thermodynamics and present equivalent law in scalable network queue. Section 5 presents the conclusion and future work. 


\section{Theory of Scalability}

Quality of service of a network system is a scalable function of the macroscopic parameters [1]. Hui proves the linear scalability of quality of service (Q) of a network queue with respect to capacity $(\mathrm{C})$, arrival rate $(\mathrm{N})$ and buffer size $(\mathrm{B})$ that is, if all the macroscopic parameters of the network queue are scaled by a constant $\alpha$ quality of service gets multiplied by the same constant. We pose this scalability property as a postulate.

$$
\mathrm{Q}(\alpha \mathrm{C}, \alpha \mathrm{N}, \alpha \mathrm{B})=\alpha \mathrm{Q}(\mathrm{C}, \mathrm{N}, \mathrm{B}) \text {. }
$$

The manifold of (Q, C, N, B) can be described by the marginals, namely taking partial derivatives using two of the four quantities while keeping the remaining two quantities constant. We study the differential form of

$$
\mathrm{Q}=\mathrm{Q}(\mathrm{C}, \mathrm{N}, \mathrm{B}) \text {. }
$$

Differentiating

$$
\begin{gathered}
d Q=\left(\frac{\partial Q}{\partial C}\right)_{B, N} d C+\left(\frac{\partial Q}{\partial B}\right)_{C, N} d B+\left(\frac{\partial Q}{\partial N}\right)_{C, B} d N \\
\left(\frac{\partial Q}{\partial C}\right)_{B, N} \equiv \theta . \\
\left(\frac{\partial Q}{\partial B}\right)_{C, N} \equiv \delta . \\
-\left(\frac{\partial Q}{\partial N}\right)_{C, B} \equiv \mu .
\end{gathered}
$$

Where $\theta, \delta, \mu$ are called microscopic/intensive parameters [1]. Hence

$$
d Q=\theta d C+\delta d B-\mu d N .
$$

Since Q is scalable in B, C, N

$$
Q=\theta C+\delta B-\mu N
$$

\section{Relation Between Microscopic Parameters}

Microscopic parameters represent the shadow price of quality of service with respect to macroscopic parameters [1]. They reflect the effect on quality of service in case the buffer size, capacity or number of sources of the system changes. 
We propose that for a scalable system it is possible to derive a relation among the intrinsic quantities independent of extensive parameters. As example we take the $\mathrm{M} / \mathrm{M} / 1 / \mathrm{B}$ and $\mathrm{M} / \mathrm{M} / \mathrm{C} / \mathrm{C}+\mathrm{B}$ queues to illustrate these relationships.

\section{A. M/M/1/B Queue}

Quality of service of $M / M / 1 / B$ [1] is

$$
\mathrm{Q}_{M / M / 1 / B}=\mathrm{B} \log \left(\frac{C}{N}\right) \text {. }
$$

Where $\mathrm{B}$ is the buffer size, $\mathrm{N}$ the total number of connections and $\mathrm{C}$ is server capacity.

$$
\begin{gathered}
\theta=\left(\frac{\partial Q}{\partial C}\right)_{N, B}=\frac{B}{C} . \\
\delta=\left(\frac{\partial Q}{\partial B}\right)_{C, N}=\log \left(\frac{C}{N}\right) . \\
\mu=\left(-\frac{\partial Q}{\partial N}\right)_{C, B}=\frac{B}{N} .
\end{gathered}
$$

From equation (10), (11) and (12)

$$
\begin{gathered}
\mu=\theta e^{\delta} . \\
\log (\mu)=\log (\theta)+\delta .
\end{gathered}
$$

Equation (14) presents relation between microscopic quantities independent of extrinsic quantities.

\section{B. $\mathrm{M} / \mathrm{M} / \mathrm{C} / \mathrm{C}+\mathrm{B}$ Queue}

Quality of service for $\mathrm{M} / \mathrm{M} / \mathrm{C} / \mathrm{C}+\mathrm{B}$ queue [1] is

$$
\mathrm{Q}_{M / M / C / C+B}=\left[\mathrm{C}\left(1-\frac{N}{C}\right)+(\mathrm{C}+\mathrm{B}) \log \rho\right]=(\mathrm{C}+\mathrm{B}) \log \left(\frac{C}{N}\right)+\mathrm{N}-\mathrm{C} \text {. }
$$

The $\mathrm{M} / \mathrm{M} / \mathrm{C} / \mathrm{C}+\mathrm{B}$ queue has $\mathrm{B}$ waiting room and $\mathrm{C}$ number of servers. Quality of service in case of a $\mathrm{M} / \mathrm{M} / \mathrm{C} / \mathrm{C}+\mathrm{B}$ queue is

$$
\begin{gathered}
\mathrm{Q}=(\mathrm{B}+\mathrm{C}) \log \left(\frac{C}{N}\right)+\mathrm{N}-\mathrm{C} . \\
\theta=\frac{B}{C}+\log \left(\frac{C}{N}\right) .
\end{gathered}
$$




$$
\begin{gathered}
\delta=\log \left(\frac{C}{N}\right) . \\
-\mu=1-\frac{B+C}{N}
\end{gathered}
$$

From equations (17), (18) and (19) we have

$$
\begin{gathered}
\mu+1=\mathrm{e}^{\delta}(\theta-\delta+1) . \\
\log (\mu+1)=\delta+\log (\theta-\delta+1) .
\end{gathered}
$$

Equation (21) presents relation between the three microscopic quantities in $\mathrm{M} / \mathrm{M} / \mathrm{C} / \mathrm{C}+\mathrm{B}$ queue. Hence we prove that microscopic parameters of a scalable network queue are related to each other independent of individual macroscopic parameters.

\section{Gibbs-Duhem Type Law In Networks}

In thermodynamics there is a relation among the intensive parameters. For a single component system $\mu$ chemical potential is a function of $\mathrm{T}$ temperature and $\mathrm{P}$ pressure.

The existence of a relationship among the various microscopic parameters is consequence of the homogenous first-order property of the fundamental relation of thermodynamics. For a single component system this property permits the fundamental relation to be written in the form $u=u(s, v)$ where $u$ is the energy per mole, $s$ is the entropy per mole and $v$ is the volume per mole; each of the three intensive parameters is then also a function of $s$ and $v$. A differential form of the relation among the intensive parameters can be obtained directly from the Euler relation and is known as the Gibbs Duhem relation proved in [2].

$$
d \mu=-s d T+v d P .
$$

The variation in chemical potential is not independent of the variation of temperature and pressure, but the variation of any one of them can be computed in the terms of the variations of the other two. Corresponding to the Gibbs Duham relation in thermodynamics we derive a Gibbs Duham type relation in scalable network queues. Microscopic quantities in a scalable system are not all independent. There is a relation among the intensive parameters and for a single component system $\mu$ is a function of $\theta$ and $\delta$. The existence of a relationship among the microscopic parameters is a consequence of homogenous first-order property of the fundamental relation. The Gibbs-Duhem relation presents relation between microscopic quantities in thermodynamics. We find the Gibbs Duhem type relation in a network queue

$$
\mathrm{Q}=\theta C+\delta B-\mu N
$$


Differentiating

$$
d \mathrm{Q}=\theta d C+\delta d B-\mu d N+\mathrm{C} d \theta+\mathrm{B} d \delta-\mathrm{N} d \mu .
$$

From equation (7)

$$
d Q=\theta d C+\delta d B-\mu d N .
$$

Subtracting equation (25) from (24)

$$
\begin{gathered}
\mathrm{N} d \mu=\mathrm{C} d \theta+\mathrm{B} d \delta . \\
d \mu=\mathrm{c} d \theta+\mathrm{b} d \delta .
\end{gathered}
$$

Where $\mathrm{b} \equiv \frac{B}{N}$ and $\mathrm{c} \equiv \frac{C}{N}$

Equation (27) represents Gibbs Duhem type of relation in scalable network queue. The variation in $\mu$ is not independent of the variations in $\theta$ and $\delta$, but the variations of any one can be computed in terms of the variations of the other two. Hence from equation (27)

$$
\begin{gathered}
\frac{\partial \mu}{\partial \theta}=c . \\
\frac{\partial \mu}{\partial \delta}=b \\
\frac{\partial \delta}{\partial \theta}=-\frac{c}{b} .
\end{gathered}
$$

Gibbs Duhem law presents relation among the microscopic parameters of the queue in the differential form. We verify these differential relationships for both the universal queues $\mathrm{M} / \mathrm{M} / 1 / \mathrm{B}$ and $\mathrm{M} / \mathrm{M} / \mathrm{C} / \mathrm{C}+\mathrm{B}$.

\section{Conclusion and Future Work}

We prove that microscopic parameters of scalable network queue are related to each other independent of macroscopic parameters of the queue. We derive equivalent Gibbs Duham law in scalable network queue. Our future work will be developing the techniques for calculating microscopic parameters and finding equivalent thermo dynamical laws applicable to any network queue.

\section{References}

[1] J.Y.Hui, Ezhan Karasan, "A Thermodynamic Theory of Broadband Networks with Applications to Dynamic Routing," IEEE J. Select. Areas Commun., vol. 13, 1995.

[2] H.B.Callen, "Thermodynamics and an Introduction to Thermostatics, second edition," John Wiley \&Sons, 1985. 